\title{
EFICIÊNCIA DO FERTILIZANTE NITROGENADO DE LIBERAÇÃO CONTROLADA NAS CARACTERÍSTICAS AGRONÔMICAS E PRODUÇÃO DE SEMENTES DO QUIABEIRO
}

\author{
Pablo Fernando Santos Alves ${ }^{1}$, Marcos Koiti Kondo², Gabriel Belfort Rodrigues ${ }^{3}$, Rodinei Facco \\ Pegoraro $^{4}$, Andréia Márcia Santos de Souza David ${ }^{5}$, Igor Santos Alves ${ }^{6}$
}

\begin{abstract}
RESUMO - A produção de sementes de quiabo em quantidade e qualidade demanda adubações nitrogenadas frequentes, cujas perdas podem ser reduzidas com fertilizantes de liberação controlada. Nesse sentido, foram avaliadas as características agronômicas e a produção de quiabo cultivar Santa Cruz 47 após a adubação nitrogenada com ureia de liberação controlada e ureia convencional. Utilizou-se o delineamento em blocos ao acaso, com quatro repetições e tratamentos arranjados em esquema fatorial $2 \times 5+2$, sendo duas fontes de nitrogênio (ureia convencional e ureia com polímero de liberação controlada) e cinco doses de adubação (48, 72, 96,120 e $144 \mathrm{~kg} \cdot \mathrm{ha}^{-1}$ ), mais dois tratamentos testemunhas (sem aplicação de nitrogênio e uma fonte de ureia com polímero de liberação controlada com $100 \%$ de ureia revestida). As variáveis analisadas foram a altura final de plantas, altura de inserção do primeiro fruto, número de frutos por planta, número de dias da antese a colheita, produtividade de sementes. O uso de ureia com polímero de liberação controlada em solos com elevado teor de matéria orgânica retarda a ocorrência do estádio fenológico de colheita dos frutos destinados à produção de sementes. A produção de frutos por plantas e a produtividade de sementes foram reduzidas com o aumento das doses de nitrogênio aplicadas.
\end{abstract}

Palavras chave: Abelmoschus esculentus, adubação nitrogenada, tecnificação na agricultura.

\section{EFFICIENCY OF CONTROLLED-RELEASE NITROGEN FERTILIZER ON AGRONOMIC CHARACTERISTICS AND SEED PRODUCTION OF OKRA}

\begin{abstract}
The quality and quantity of okra seed production demand much nitrogen fertilization, whose losses can be decrease with controlled-released sources. In this sense, we evaluated agronomic characteristics and yield of okra cultivar Santa Cruz 47 after nitrogen fertilization with controlled-released and conventional urea. A randomized block design was used with four replications and $2 \times 5+2$ factorial arrangement, involving two nitrogen sources (conventional and controlled-released urea) and five nitrogen levels (48, 72, 96, 120 and $144 \mathrm{~kg} \cdot \mathrm{ha}^{-1}$ ), more two control treatments (without nitrogen application and a 100\% controlled-released urea). We analyzed final plant height, insertion height of first fruit, number of fruits per plant, number of
\end{abstract}

\footnotetext{
${ }^{1}$ Discente do Programa de Produção Vegetal no Semiárido, Departamento de Ciências Agrárias, Universidade Estadual de Montes Claros (UNIMONTES), Campus Janaúba, Av. Reinaldo Viana, 2630, Caixa Postal 91, Bico da Pedra, CEP $39440-$ 000, Janaúba, MG, agrotecnico10@yahoo.com.br, Bolsista CAPES;

${ }^{2}$ Professor, Departamento de Ciências Agrárias, UNIMONTES, Campus Janaúba, Av. Reinaldo Viana, 2630, Caixa postal 91, Bico da Pedra, CEP 39440-000, marcos.kondo@unimontes.br;

${ }^{3}$ Engenheiro Agrônomo, D.Sc., Qualihort Sementes Ltda, Perímetro Irrigado do Gorutuba, Lote E 104, S/N, Zona Rural, CEP 39525-000, Nova Porteirinha, MG, gabelfort@yahoo.com;

${ }^{4}$ Professor, Instituto de Ciências Agrárias - ICA, UFMG, Avenida Universitária, 1.000 - Bairro Universitário/Montes Claros - MG, rodinei_pegoraro@yahoo.com.br;

${ }^{5}$ Professora, Departamento de Ciências Agrárias, UNIMONTES, Campus Janaúba, Av. Reinaldo Viana, 2630, Caixa postal 91, Bico da Pedra, CEP 39440-000, andreia.david@unimontes.br;

${ }^{6}$ Engenheiro agrônomo, M.Sc., Programa de Produção Vegetal, Universidade Federal dos Vales do Jequitinhonha e Mucuri, igors.agro@gmail.com.
} 
days from anthesis to harvest and seed production. The use of controlled-release polymer urea in soils with high organic matter delay the occurrence of the phenological stage of harvesting the fruits destined to seed production. The production of seed and fruits per plant decreased with increment of nitrogen doses.

Keywords: Abelmoschus esculentus, nitrogen fertilization, technicization in agriculture.

\section{INTRODUÇÃO}

A recomendação de fertilizantes para a cultura do quiabeiro destinada à produção de sementes é geralmente semelhante àquela utilizada para a produção de frutos para consumo in natura. No entanto, a colheita de frutos para consumo humano é feita quando os mesmos encontram-se imaturos, ou seja, na sua maturidade hortícola (Mota et al., 2005). Sendo assim, essas recomendações não enfatizam o potencial da adubação sobre o desenvolvimento da cultura de modo a maximizar a produtividade e a qualidade das sementes, uma vez que a colheita dos frutos para a produção de sementes deve ser realizada após a maturidade fisiológica das sementes.

Com relação à nutrição mineral da cultura do quiabeiro, Filgueira (2008) considera o nitrogênio como o nutriente mais exigido. Este elemento está intimamente ligado à produção de proteínas, que são constituintes importantes no desenvolvimento inicial do embrião durante a germinação das sementes. Além disso, o nitrogênio interfere em diversas outras características das plantas relacionadas ao crescimento e desenvolvimento, as quais, direta ou indiretamente, afetam a produtividade de sementes (Oliveira et al., 2006; Barbosa et al., 2011).

Sabe-se que o $\mathrm{N}$ tem alto dinamismo no solo, sofrendo diversas transformações químicas e biológicas. Devido ao seu baixo efeito residual, e sua alta exigência pelas culturas, a adubação nitrogenada é feita em maior quantidade, e com mais frequência que os demais nutrientes (D’Andrea et al., 2004).

A estratégia mais comum para minimizar as perdas é adequar a aplicação do fertilizante nitrogenado às necessidades da cultura, levando em conta as características do produto usado (Cantarella et al., 2008). No entanto, nem sempre essa adequação é viável do ponto de vista agronômico, pois geram custos operacionais adicionais e também quando a aplicação possui alguma limitação, como o plantio das culturas em espaçamentos reduzidos, a falta de máquinas e implementos apropriados, a escassez ou custo elevado de mão de obra, as condições topográficas do terreno e a umidade do solo.

A tendência mundial do agronegócio nas últimas décadas esteve direcionada para a intensificação da produção agrícola. E um dos grandes desafios é buscar alternativas para produzir alimentos por meio da otimização do uso de insumos agrícolas, possibilitando ganhos econômicos para o agricultor e reduzindo o impacto ambiental da atividade (Yamada \& Abdalla, 2007).

Neste sentido, surge como alternativa de redução das perdas de nitrogênio no sistema solo-plantaatmosfera a utilização dos fertilizantes de liberação controlada ou liberação lenta, entre outras denominações. As principais vantagens desses fertilizantes seriam: fornecimento regular e contínuo de nutrientes na época necessária para as plantas; menor frequência de aplicações; redução de perdas de nutriente por lixiviação, desnitrificação, imobilização e volatilização; eliminação de danos causados às sementes e raízes devido à alta concentração de sais; maior praticidade no manuseio dos fertilizantes; redução da poluição ambiental pelo nitrato $\left(\mathrm{NO}_{3}\right)$, atribuindo valor ecológico à atividade agrícola, além da redução nos custos de produção (Shaviv, 2001).

Havendo poucos estudos que comprovem a eficiência desses adubos, o presente trabalho teve como objetivo avaliar as características agronômicas, a produção e qualidade de sementes de quiabo cultivar Santa Cruz 47 após a adubação nitrogenada, utilizando ureia com polímero de liberação controlada e ureia convencional.

\section{MATERIAL E MÉTODOS}

O experimento foi conduzido em uma área da empresa QUALIHORT SEMENTES LTDA, localizada no município de Nova Porteirinha, MG, no Perímetro Irrigado do Gorutuba, apresentando latitude $15^{\circ} 48^{\prime} 09^{\prime \prime}$ $\mathrm{S}$ e longitude $43^{\circ} 18^{\prime} 32^{\prime \prime} \mathrm{O}$, com altitude de $533 \mathrm{~m}$. O solo foi classificado como Cambissolo Háplico, EMBRAPA (2013). O clima, segundo Köppen, é do tipo 
AW (tropical chuvoso, savana com inverno seco). O experimento ocorreu durante os meses de dezembro de 2011 a maio de 2012.

Os dados climatológicos referentes a esse período encontram-se na Figura 1.

O solo do experimento apresenta elevada fertilidade, caracterizada pelos resultados das análises do solo (0-20 cm de profundidade), antes da implantação do experimento, que apresentaram os seguintes valores: $\mathrm{pH}$ em $\mathrm{H}_{2} \mathrm{O}(6,9) ; \mathrm{H}+\mathrm{Al}\left(1,25 \mathrm{cmol}_{\mathrm{c}} \cdot \mathrm{dm}^{-3}\right) ; \mathrm{Al}(0,0$ $\left.\mathrm{cmol}_{\mathrm{c}} \cdot \mathrm{dm}^{-3}\right) ; \mathrm{Ca}\left(10,0 \mathrm{cmol}_{\mathrm{c}} \cdot \mathrm{dm}^{-3}\right) ; \mathrm{Mg}\left(3,8 \mathrm{cmol}_{\mathrm{c}} \cdot \mathrm{dm}^{-3}\right)$; $\mathrm{K}\left(404,0 \mathrm{mg} \cdot \mathrm{dm}^{-3}\right) ; \mathrm{P}\left(124 \mathrm{mg} \cdot \mathrm{dm}^{-3}\right) ; \mathrm{Zn}\left(74,8 \mathrm{mg} \cdot \mathrm{dm}^{-3}\right)$; $\mathrm{Fe}\left(21,6 \mathrm{mg} \cdot \mathrm{dm}^{-3}\right) ; \mathrm{Mn}\left(111,1 \mathrm{mg} \cdot \mathrm{dm}^{-3}\right) ; \mathrm{Cu}\left(0,5 \mathrm{mg} \cdot \mathrm{dm}^{-3}\right)$; Matéria orgânica $\left(7,7\right.$ dag. $\left.\mathrm{kg}^{-1}\right)$; SB $\left(14,9 \mathrm{cmol}_{\mathrm{c}} \cdot \mathrm{dm}^{-3}\right)$; $\mathrm{T}\left(16,1 \mathrm{cmol}_{\mathrm{c}} \cdot \mathrm{dm}^{-3}\right) ; \mathrm{t}\left(14,9 \mathrm{cmol}_{\mathrm{c}} \cdot \mathrm{dm}^{-3}\right) ; \mathrm{V}(92,2 \%)$; m (0,0\%); Areia (53 dag. $\left.\mathrm{kg}^{-1}\right)$; Silte (34 dag. $\left.\mathrm{kg}^{-1}\right)$; Argila (13 dag. $\left.\mathrm{kg}^{-1}\right)$; Classe textural franca.

Devido às condições planas e agricultáveis da área, não foi realizado o revolvimento do solo por meio da aração ou gradagem. Dessa forma, o preparo do solo constituiu na eliminação de plantas daninhas, escarificação manual com o auxílio de enxada e o sulcamento.

Não foi necessária a aplicação de corretivo. Aplicouse $40 \mathrm{~kg} \cdot$ ha $^{-1} \mathrm{de}_{2} \mathrm{O}_{5}$ (superfosfato simples, na semeadura) e $60 \mathrm{~kg} \cdot \mathrm{ha}^{-1} \mathrm{de}_{2} \mathrm{O}(\mathrm{KCl}, 40 \%$ na semeadura e três coberturas iguais, aos 20, 40 e 60 DAE) (Correia et al., 1999). Todos os fertilizantes, na semeadura, foram aplicados no sulco, abaixo e ao lado das sementes.

Utilizou-se o delineamento fatorial ( 2 fontes $\mathrm{x} 5$ doses +2 tratamentos adicionais), em blocos

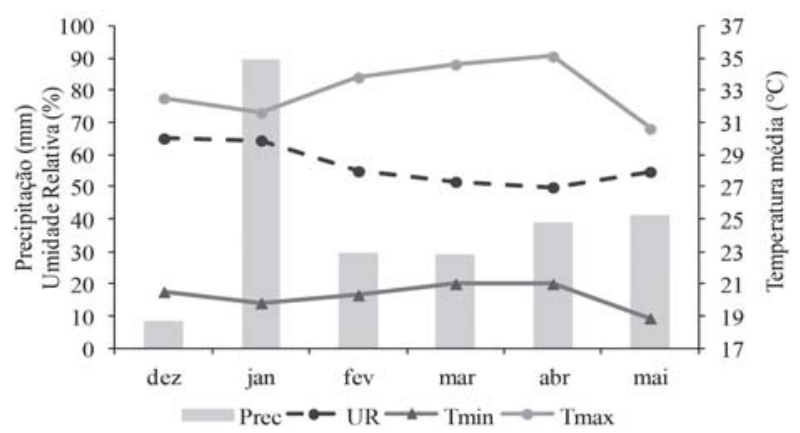

Figura 1 - Precipitação (Prec), umidade relativa do ar (UR), temperatura média mínima (Tmin), temperatura média máxima (Tmax) no período de condução do experimento. casualizados com 4 repetições. As fontes testadas foram: 1) ureia convencional (UC) ( $20 \%$ no plantio e $80 \%$ em três coberturas: 20, 40 e $60 \mathrm{DAE})$; 2) Ureia com polímero de liberação controlada (UPLC) (constituída de ureia convencional (30\%) mais ureia com polímero de liberação controlada (70\%), contendo $40,45 \%$ de N) aplicada toda no plantio. As doses testadas foram: 48, 72, 96, 120 e $144 \mathrm{~kg} \cdot \mathrm{ha}^{-1} \mathrm{de}$ N. Os tratamentos adicionais ao fatorial foram o $\mathrm{T}_{0}$ (testemunha, sem adubação nitrogenada) e o $\mathrm{T}_{1}$ (ureia com polímero de liberação controlada com $38,5 \%$ de N) na dose de $120 \mathrm{~kg} \cdot \mathrm{ha}^{-1}$, aplicada toda no plantio.

A semeadura foi em 23 de dezembro de $2011 \mathrm{com}$ três sementes tratadas do cultivar Santa Cruz 47 por cova. Posteriormente manteve-se somente uma planta por cova, com $0,2 \mathrm{~m}$ entre plantas e $0,8 \mathrm{~m}$ entre linhas de covas. Das parcelas com 16 plantas (4 linhas x 4 plantas.linha $\left.{ }^{-1}\right)$, utilizaram-se as quatro plantas centrais. Os tratos culturais seguiram as recomendações para a cultura.

A partir da emissão dos primeiros botões florais, verificou-se diariamente a fase em que $50 \%$ das plantas das parcelas tiveram pelo menos uma flor totalmente aberta (antese). O período compreendido entre a antese e a colheita (DAC) foi contabilizado, sendo considerada, nesse caso, a primeira colheita em cada parcela. A colheita dos frutos aconteceu quando apresentaram a coloração amarelo-palha, indicando a maturação físiológica das sementes (Setubal et al., 1994). No período em que se iniciou a colheita, foram avaliadas também a altura final de plantas (ALFP) e altura de inserção do primeiro fruto (ALPF).

Os frutos foram analisados no Laboratório de Análise de Sementes, onde se determinou o número de frutos por planta (FP). Após a extração manual, as sementes foram armazenadas em sacos de papel até atingirem equilíbrio higroscópico, sendo posteriormente determinadas a produtividade de sementes (PROD) e o peso de mil sementes (PMS) (BRASIL, 2009). Para isso, foi realizada previamente a correção da umidade para $13 \%$.

As características avaliadas foram submetidas à análise de variância (teste F). Quando constatada diferença estatística entre os tratamentos, atribuída ao efeito de diferentes doses de nitrogênio, realizouse a análise de regressão. Para a comparação das médias de tratamento em relação às testemunhas aplicou-se 
o teste de Dunnet a 5\% de probabilidade. Os dados foram analisados por meio do software estatístico $\mathrm{R}$ (R Core Team, 2016).

\section{RESULTADOS E DISCUSSÃO}

As variáveis altura final de plantas (ALFP) e altura de inserção do primeiro fruto (ALPF) não foram influenciadas pelos fatores estudados (Tabela 1).

Com relação à variável número de dias da antese a colheita (DAC), é possível observar na Tabela 1 que os tratamentos que receberam ureia com polímero apresentaram média de DAC superior em cinco dias aos tratamentos que receberam ureia convencional. O momento ideal para a colheita dos frutos de quiabeiro para a produção de sementes com alto potencial fisiológico tem sido caracterizado pelo aumento da relação carbono:nitrogênio nos mesmos, situação evidenciada pela mudança de tonalidade verde para a tonalidade amarelo-palha a marrom-clara, sem que ainda o pedúnculo esteja seco. Desta maneira, por liberar de maneira gradual o nitrogênio para as plantas, o uso da UPLC diminui as perdas por volatilização, tendo como consequência uma possível ocorrência de valores mais baixos da relação carbono:nitrogênio nos frutos, retardando a colheita.

A recomendação de calagem e adubação para as culturas é geralmente efetuada com base nas doses de corretivos e fertilizantes que assegurem entre $80 \%$ e $90 \%$ de produção relativa, equivalente à produção de máxima eficiência econômica (nível crítico). Isso, no entanto, nem sempre é válido, considerando que a máxima eficiência econômica é inerente ao valor econômico do produto e à eficiência econômica do sistema de produção (Cantarutti et al., 2007). Na Figura 2, observa-se efeito quadrático na produção de frutos por planta em função da adubação nitrogenada.
Considerando que o melhor efeito agronômico seja o maior número de frutos por planta, estima-se que a melhor dose de nitrogênio a ser recomendada seja a dose de 77,2 kg.ha-1 ${ }^{-1}$. No entanto, é possível observar na Tabela 2 que nenhuma combinação de fonte e dose de nitrogênio testada superou a produção obtida com o tratamento testemunha $\mathrm{T}_{0}$ (sem adubação nitrogenada).

A diminuição do número de frutos por planta apresentou reflexo similar na produtividade de sementes (PROD), de maneira que a aplicação de doses de nitrogênio além do nível de 73,9 kg.ha ${ }^{-1}$ propiciou uma redução acentuada da produtividade de sementes (Figura 3 ). Este fato fica mais evidenciado nos tratamentos provenientes da combinação de ureia convencional e os níveis de 120 e 144 kg.ha-1 , os quais diferiram da testemunha $\left(\mathrm{T}_{0}\right)$, com reduções de 45,8 e $54,6 \%$, respectivamente (Tabela 2 ). A redução da produtividade

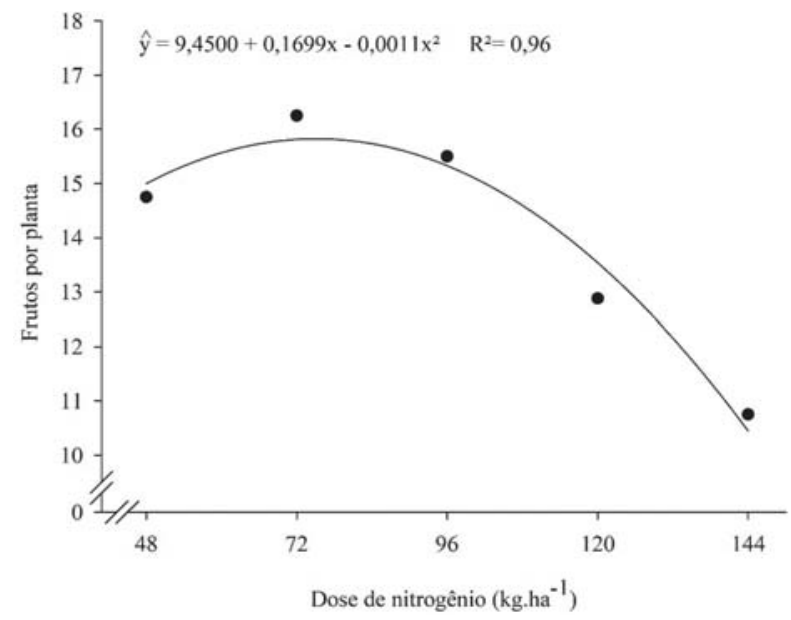

Figura 2 - Número de frutos por planta (FP) em quiabeiro cultivar Santa Cruz 47 em função da dose de nitrogênio aplicada.

Tabela 1 - Valores médios e teste F para as variáveis: altura de plantas (ALFP), altura de inserção do primeiro fruto (ALPF), número de dias da antese a colheita (DAC), número de frutos por planta (FP), produtividade de sementes (PROD), peso de mil sementes (PMS) em quiabeiro cultivar Santa Cruz 47

\begin{tabular}{|c|c|c|c|c|c|c|}
\hline \multirow{2}{*}{ Causa da variação } & \multicolumn{6}{|c|}{ Valores médios } \\
\hline & $\begin{array}{c}\text { ALFP } \\
(\mathrm{cm})\end{array}$ & $\begin{array}{l}\text { ALPF } \\
(\mathrm{cm})\end{array}$ & $\begin{array}{c}\text { DAC } \\
\text { (dias) }\end{array}$ & FP & $\begin{array}{l}\text { PROD } \\
\left(\mathrm{kg}^{\circ} \mathrm{ha}^{-1}\right)\end{array}$ & $\begin{array}{c}\text { PMS } \\
(\mathrm{g})\end{array}$ \\
\hline \multicolumn{7}{|c|}{ Fonte de nitrogênio (F) } \\
\hline UPLC & $214 \mathrm{a}$ & 96 a & $45 \mathrm{a}$ & $14 \mathrm{a}$ & 3340 a & 55,0 a \\
\hline $\mathrm{UC}$ & $211 \mathrm{a}$ & $96 \mathrm{a}$ & $40 \mathrm{~b}$ & $14 \mathrm{a}$ & 3216 a & 55,3 a \\
\hline
\end{tabular}

Médias seguidas de uma mesma letra na coluna não diferiram estatisticamente pelo teste $\mathrm{F}(\mathrm{p}<0,05)$. 
Tabela 2 - Valores médios das variáveis: altura de plantas (ALFP), altura de inserção do primeiro fruto (ALPF), número de dias da antese a colheita (DAC), número de frutos por planta (FP), produtividade de sementes (PROD), peso de mil sementes (PMS) em quiabeiro cultivar Santa Cruz 47.

\begin{tabular}{|c|c|c|c|c|c|c|}
\hline Tratamentos & $\operatorname{ALFP}(\mathrm{cm})$ & ALPF $(\mathrm{cm})$ & DAC (dias) & FP & PROD (kg.ha-1) & $\operatorname{PMS}(\mathrm{g})$ \\
\hline UPLC 48 & 207 & 94 & 44 & 15 & 3695 & 55,4 \\
\hline UPLC 72 & 237 & 94 & 42 & 15 & 3068 & 55,5 \\
\hline UPLC 96 & 203 & 97 & 44 & 15 & 3708 & 56,8 \\
\hline UPLC 120 & 214 & 111 & 47 & 14 & 3324 & 52,7 \\
\hline UPLC 144 & 210 & 82 & 46 & 12 & 2907 & 54,5 \\
\hline $\mathrm{UC} 48$ & 226 & 98 & 35 & 14 & 3303 & 55,0 \\
\hline UC 72 & 213 & 89 & 38 & 17 & 4264 & 56,6 \\
\hline UC 96 & 198 & 94 & 44 & 16 & 3737 & 57,5 \\
\hline UC 120 & 218 & 96 & 38 & 11 & $2599 *$ & 54,1 \\
\hline UC 144 & 203 & 105 & 45 & $10 *$ & $2176 *$ & 53,1 \\
\hline Testemunha $1\left(\mathrm{~T}_{1}\right)$ & 216 & 105 & 43 & 13 & 2964 & 55,6 \\
\hline Testemunha $0\left(\mathrm{~T}_{0}\right)$ & 229 & 90 & 38 & 17 & 4793 & 58,1 \\
\hline
\end{tabular}

Médias seguidas de asterisco $(*)$ diferiram estatisticamente da testemunha zero $\left(\mathrm{T}_{0}\right)$ pelo teste de Dunnett $(\mathrm{P}<0,05)$.

de sementes em função da realização da adubação nitrogenada foi notada também entre as testemunhas $\mathrm{T}_{0}$ (sem adubação nitrogenada) e $\mathrm{T}_{1}\left(120 \mathrm{~kg} \cdot \mathrm{ha}^{-1} \mathrm{de}\right.$ nitrogênio no plantio utilizando $100 \%$ de ureia revestida) (Tabela 1).

Segundo Duarte \& Peil (2010) o avantajado porte da planta provocado pelo excessivo crescimento vegetativo ocasiona autossombreamento é prejudicial à taxa fotossintética individual de cada planta, diminuindo assim a disponibilidade de fotoassimilados para as mesmas. Sendo assim, a aplicação de nitrogênio em doses excessivas, independente da fonte utilizada, levou as plantas à vegetação, diminuindo o número de estruturas reprodutivas com efeito direto na produtividade de sementes, o que corrobora com outros trabalhos na literatura científica (Qiao et al., 2012).

Lopes \& Sa (2009) trabalhando com a cultivar Santa Cruz 47 observaram que o aumento da dose de nitrogênio utilizada proporcionou aumento linear do número de frutos de quiabo por planta, no entanto, doses maiores que $42 \mathrm{~kg} \cdot \mathrm{ha}^{-1}$ de nitrogênio ocasionavam redução da produtividade, de maneira semelhante ao que foi constatado no presente trabalho. Okumura et al. (2013), avaliando a eficiência no uso da ureia convencional e ureia revestida com inibidor de urease na cultura do milho verificaram que os níveis crescentes de $\mathrm{N}$, independentemente da fonte utilizada, resultaram em uma diminuição dos ganhos de eficiência agronômica e fisiológica do nutriente.
Qiao et al. (2012) avaliando a redução da adubação nitrogenada na cultura do arroz por dois anos consecutivos, verificaram que elevadas doses de nitrogênio reduziram a eficiência de uso no nutriente, sendo que deduções de até $14 \%$ em relação as doses comumente utilizadas pelos produtores não afetavam o rendimento de grãos. Além disso, a redução da adubação nitrogenada pode diminuir os efeitos nocivos do $\mathrm{N}$ em excesso no ambiente (Qiao et al., 2013).

De acordo Galati et al. (2013) a quantidade e a proporcionalidade dos nutrientes absorvidos pelas plantas são funções de características intrínsecas do vegetal, como, também, dos fatores externos que influenciam tanto a disponibilidade dos nutrientes como a absorção destes pelas raízes e, consequentemente, o crescimento da parte aérea e produtividade das culturas. Dessa maneira, a mineralização da matéria orgânica, em alto conteúdo no solo da área experimental, supriu adequadamente a demanda da cultura por nitrogênio, tendo sido potencializada pelas temperaturas elevadas e pela manutenção da umidade do solo próximo à capacidade de campo. Além disso, é possível que o alto teor de nitrogênio disponível no solo tenha influenciado negativamente a atuação de microrganismos benéficos ao quiabeiro, assim como demonstrado em outras culturas (Waterer \& Vessey, 1993; Xavier et al., 2008; Martins et al., 2013), explicando em parte o efeito negativo na produção de sementes. 
Apesar dos efeitos proporcionados pelo excesso da adubação nitrogenada na redução da produtividade de sementes, observa-se que a translocação de fotoassimilados para a formação e ganho de peso das sementes, expresso pelo peso de mil sementes (PMS), não foi influenciado pelos fatores estudados (Tabelas 1 e 2).

\section{CONCLUSÕES}

O uso de ureia com polímero de liberação controlada em solos com elevado teor de matéria orgânica retarda a ocorrência do estádio fenológico de colheita dos frutos destinados à produção de sementes, o que sugere nessas condições a possibilidade de redução das doses a serem utilizadas, tendo em vista a antecipação da colheita.

Nas condições deste estudo, a adubação nitrogenada em dose acima de 77,2 e 73,9 kg.ha- ${ }^{-1}$ reduz o número de frutos por planta e a produtividade de sementes do quiabeiro, respectivamente.

\section{LITERATURA CITADA}

BARBOSA, R. M.; COSTA, D. S.; HOMEM, B. F. M.; SÁ, M. E. Nitrogênio na produção e qualidade de sementes de feijão. Pesquisa Agropecuária Tropical, v. 41, n. 3, p. 470474, 2011. Disponível em: http://dx.doi.org/ 10.5216/pat.v41i3.12761 (Acesso em 22/11/2017)

BRASIL. Ministério da Agricultura, Pecuária e Abastecimento. Regras para análise de sementes. Brasília: SNAD/DNDV/CLAV. 365 p, 2009.

CANTARELLA, H.; TRIVELIN, P. C. O.; CONTIN, T. L. M.; DIAS, F. L. F.; ROSSETTO, R.; MARCELINO, R.; COIMBRA, R. B.; QUAGGIO, J. A. Ammonia volatilisation from urease inhibitortreated urea applied to sugarcane trash blankets. Scientia Agricola, v.65, p.397-401, 2008. Disponível em: http://dx.doi.org/10.1590/S010390162008000400011 (Acesso em 22/11/2017).

CANTARUTTI, R. B.; BARROS, N. F.; MARTINEZ, H. E. P.; NOVAIS, R. F. Avaliação da fertilidade de solo e recomendação de fertilizantes. In: NOVAIS, R. F.; ALVAREZ V., V. H.; BARROS, N. F.; FONTES, R. L. F.; CANTARUTTI, R. B.; NEVES, J. C. L. (Ed.). Fertilidade do solo. Viçosa: SBCS. p.769-850, 2007.
CORREIA, L. G.; FILHO, J. A. A.; NAGAI, H. Sugestões de adubação para hortaliças - Quiabo.

In: Recomendações para o uso de corretivos e fertilizantes em Minas Gerais (5 ${ }^{\mathrm{a}}$ APROXIMAÇÃO). Comissão de Fertilidade do Solo do Estado de Minas Gerais CFSEMG.Viçosa-MG, 1999. 202p.

D’ANDRÉA, A. F.; SILVA, M. L. N.; CURI, N.; GUILHERME, L. R. G. Estoque de carbono e nitrogênio e formas de nitrogênio mineral em um solo submetido a diferentes sistemas de manejo. Pesquisa Agropecuária Brasileira, v. 39, n. 2, p. 179-186, 2004. Disponível em: http:// dx.doi.org/10.1590/S0100-204X2004000200012 (Acesso em 22/11/2017).

DUARTE, T.S; PEIL, R.M.N. Relações fonte:dreno e crescimento vegetativo do meloeiro.

Horticultura Brasileira, v. 28, n.3, p. 271-2, 2010. Disponível em: http://dx.doi.org/10.1590/S010205362010000300005 (Acesso em 22/11/2017).

EMPRESA BRASILEIRA DE PESQUISA AGROPECUÁRIA - EMBRAPA. Sistema brasileiro de classificação de solos. 3. ed. Rio de janeiro. 2013.353p.

FILGUEIRA, F. A. R. Novo manual de olericultura: agrotecnologia moderna na produção e comercialização de hortaliças. 3o ed. Viçosa-MG: Editora UFV. 2008. 421p.

GALATI, V. C.; CECÍLIO FILHO, A. B.; GALATI, V. C.; ALVES, A. U. Crescimento e acúmulo de nutrientes da cultura do quiabeiro. Semina: Ciências Agrárias, v.34, n.1, p.191-200, 2013. Disponível em: http://dx.doi.org/10.5433/16790359.2013v34n1p191 (Acesso em 22/11/2017).

LOPES, A.W.P.; SA, M.E. Doses e épocas da adubação nitrogenada e poda apical na produção e qualidade das sementes de quiabeiro. Cultura Agronômica, v.17, p.51-56, 2009.

MARTINS, R. N. L.; NÓBREGA, R. S. A.; SILVA, A. F. T.; NÓBREGA, J. C. A.; AMARAL, F. H. C.; COSTA, E. M.; LUSTOSA FILHO, J. F.; MARTINS, L. V. Nitrogênio e micronutrientes na produção de grãos de feijão-caupi inoculado. Semina:

Ciências Agrárias, v.34, n.4, p.1577-1586, 2013. Disponível em: http://dx.doi.org/10.5433/16790359.2013v34n4p1577 (Acesso em 22/11/2017). 
MOTA, W. F.; FINGER, F. L.; SILVA, D. J. H.; CORRÊA, P. C.; FIRME, L. P.; NEVES, L. L. DE M. Caracterização físico-química de frutos de quatro cultivares de quiabo. Horticultura brasileira, v.23, n.3, p.722-725, 2005. Disponível em: http://dx.doi.org/10.1590/S010205362005000300006 (Acesso em 22/11/2017).

OKUMURA, R.; MARIANO, D.C.; ZACCHEO, P.; ALBUQUERQUE, A. de; GIEBELMEIER, C.; LOBATO, A.K. da S.; FRANCO, A.; OLIVEIRA, N. de; SALDANHA, E.; CONCEIÇÃO, H. da. Efficiency of utilization of nitrogen coated with urease inhibitor in maize. Pakistan journal of biological sciences: PJBS, v.16, n.17, p.871-876, 2013. Disponível em: http://dx.doi.org/ 10.3923/pjbs.2013.871.876 (Acesso em 22/11/2017).

OLIVEIRA, A. P. de; ALVES, E. U.; BRUNO, R. de L.A.; SADER, R.; ALVES, A. U. Produção e qualidade fisiológica de sementes de coentro em função de doses de nitrogênio. Revista

Brasileira de Sementes, v. 28, p. 193-198, 2006. Disponível em: http://dx.doi.org/10.1590/ S0101-31222006000100027 (Acesso em 22/11/2017).

QIAO, J.; YANG, L.; YAN, T.; XUE, F.; ZHAO, D. Nitrogen fertilizer reduction in rice production for two consecutive years in the Taihu Lake area.

Agriculture, Ecosystems \& Environment, v.146, n.1, p.103-112, 2012. Disponível em: http://dx.doi.org/10.1016/ j.agee.2011.10.014 (Acesso em 22/11/2017).

QIAO, J.; YANG, L.; YAN, T.; XUE, F.; ZHAO, D. Rice dry matter and nitrogen accumulation, soil mineral $\mathrm{N}$ around root and $\mathrm{N}$ leaching, with increasing application rates of fertilizer.

European Journal of Agronomy, v. 49, p. 93-103, 2013. Disponível em: http://dx.doi.org/ 10.1016/j.eja.2013.01.009 (Acesso em 22/11/2017).
R CORE TEAM. R: A language and environment for statistical computing. R Foundation for Statistical Computing, Vienna, Austria, 2016. Disponível em: https://www.Rproject.org/. (Acesso em 22/11/2017)

SETUBAL, J.W.; ZANIN, A.C.W.; NAKAGAWA, J. Efeitos de métodos de colheita e da localização dos frutos na planta sobre a ocorrência de sementes duras em quiabeiro (Abelmoschus esculentus (L) Moench). Scientia Agrícola, v.51, n.3, p.490-493,1994. Disponível em: http:// dx.doi.org/10.1590/S0103-90161994000300019 (Acesso em 22/11/2017).

SHAVIV, A. Advances in controlled-release fertilizers. Advances in Agronomy, San Diego, v.71, p.1-49, 2001. Disponível em: http:// dx.doi.org/10.1016/S0065-2113(01)71011-5 (Acesso em 22/11/2017).

WATERER, J. G.; VESSEY, J. K. Effect of low static nitrate concentrations on mineral nitrogen uptake, nodulation, and nitrogen fixation in field pea. Journal of Plant Nutrition, v.16, n.9, p.1775-1789, 1993. Disponível em: http:// dx.doi.org/10.1080/01904169309364649 (Acesso em 22/11/2017).

XAVIER, T.F.; ARAÚJO, A.S.F. de; SANTOS, V.B. dos; CAMPOS, F.L. Inoculação e adubação nitrogenada sobre a nodulação e a produtividade de grãos de feijão-caupi. Ciência Rural, v.38, n.7, p.2037-2041, 2008. Disponível em: http:// dx.doi.org/10.1590/S0103-84782008000700038 (Acesso em 22/11/2017).

YAMADA, T.; ABDALLA, S.R.S. Informações recentes para otimização da produção agrícola. In: International Plant Nutrition Institute - IPNI. Informações Agronômicas. Nº117, 2007.

Recebido para publicação em 22/11/2017 e aprovado em 8/6/2018. 